\title{
Properties of Dye Sensitized Solar Cells with Adding Nano Carbon Black into Blocking Layer
}

\author{
Kwangbae Kim, Yunyoung Noh, and Ohsung Song ${ }^{\dagger}$ \\ Department of Materials Science and Engineering, University of Seoul, Seoul 130-743, Korea \\ (Received March 25, 2015; Revised May 26, 2015; Accepted May 27, 2015)
}

\begin{abstract}
Blocking layers with nano carbon blacks $(\mathrm{NCBs})$ were prepared by adding $0.0 \sim 0.5 \mathrm{wt} \% \mathrm{NCBs}$ to the $\mathrm{TiO}_{2}$ blocking layer. Then, dye sensitized solar cells (DSSCs) were fabricated with a $0.45 \mathrm{~cm}^{2}$ active area. TEM and micro-Raman spectroscopy were used to characterize the microstructure and phases of the NCBs, respectively. Optical microscopy and AFM were used to analyze the microstructure of the $\mathrm{TiO}_{2}$ blocking layer with NCBs. UV-VIS-NIS spectroscopy was used to determine the band gap of the $\mathrm{TiO}_{2}$ blocking layer with NCBs. A solar simulator and potentiostat were used to determine the photovoltaic properties and impedance of DSSCs with NCBs. The energy conversion efficiency (ECE) increased from 3.53 to $6.20 \%$ when the NCB content increased from 0.0 to $0.3 \mathrm{wt} \%$. This indicates that the effective surface area and electron mobility increased in the $\mathrm{TiO}_{2}$ blocking layer with NCBs. However, the ECE decreased when the NCB content was increased to over $0.4 \mathrm{wt} \%$. This change occurred because the effective electron transport area decreased with the addition of excessive $\mathrm{NCBs}$ to the $\mathrm{TiO}_{2}$ blocking layer. The results of this study suggest that the ECE of DSSCs can be enhanced by adding the appropriate amount of $\mathrm{NCBs}$ to the TiO 2 blocking layer.
\end{abstract}

Key words : Nano carbon black, Blocking layer, Energy conversion efficiency, Dye sensitized solar cell

\section{Introduction}

D ye sensitized solar cells (DSSCs) have been studied intensively as a next-generation energy device since it was first introduced in 1991 by O'Regan and M. Grätzel. ${ }^{1)}$ The new cell can provide low-cost and ease of fabrication into larger diameters, compared to the conventional silicon cell.

A DSSC typically consists of working electrode, electrolyte, and counter electrode to perform a redox reaction. Light enters the cell through a transparent electrode, and is absorbed by sensitizer generating an excited electron-hole pair. Then the electron can move within the conduction band of a neighboring $\mathrm{TiO}_{2}$ semiconductor (SC). Current is generated when the transported electron moves to working electrode through interfaces of nano $\mathrm{TiO}_{2}$. The generated hole in dye material, on the other hand, moves to counter electrode through the electrolyte, and is reduced by accepting an electron. ${ }^{2}$

The working electrode consists of transparent conductive oxide (TCO), blocking layer (compact $\mathrm{TiO}_{2}$ ), oxide SC layer (mesoporous $\mathrm{TiO}_{2}$ ), and dye. To increase efficiency of DSSC, studies have been mostly involved in engineering the working electrode, especially the oxide SC layer. Kilic et $a l .{ }^{3)}$ reported an increase in energy conversion efficiency (ECE)

${ }^{\dagger}$ Corresponding author: Ohsung Song

E-mail : songos@uos.ac.kr

Tel : +82-2-6490-2410 Fax : +82-2-6490-2404 up to $7.27 \%$ from $5.10 \%$ by adding $\mathrm{Fe}_{2} \mathrm{O}_{3}$ into $\mathrm{TiO}_{2} \mathrm{SC}$, and thus decreasing the number of recombining electrons via band gap engineering.

Zhang et $a l .{ }^{4)}$ accomplished $6.34 \%$ of ECE from $3.63 \%$ by incorporating carbon nano tube $(\mathrm{CNT})$ into $\mathrm{TiO}_{2} \mathrm{SC}$, and thus by increasing its specific surface area and electron mobility. Kim et al. ${ }^{5)}$ reduced potential barrier between TCO and $\mathrm{TiO}_{2} \mathrm{SC}$ by adding porous nano carbon into $\mathrm{TiO}_{2} \mathrm{SC}$, and achieved $3.38 \%$ of ECE compared to $2.49 \%$ of the unloaded $\mathrm{TiO}_{2} \mathrm{SC}$.

On the other hand, engineering on blocking layer is relatively less studied. However, Noh et al. ${ }^{6}{ }^{6}$ studied on the nano-diamonds (NDs) added blocking layer, and reported an increase in ECE recently. They proposed that the increased specific surface area of blocking layer led an increase in both the amount of absorbed dye on $\mathrm{TiO}_{2} \mathrm{SC}$ and the shunt resistance of $\mathrm{TiO}_{2}$ blocking layer. The result suggests that incorporation of other carbon sources into blocking layer could also enhance ECE of DSSCs. Note that nano carbon black (NCB) has bonding of mainly sp2-orbital, like many other allotropes such as graphite, graphene, CNT, and $\mathrm{ND}^{7,8)}$. Also, note that analysis of these allotropes in NCB can be conveniently carried out by Raman spectroscopy. $^{9-11)}$

We expected that NCB can effectively increase the specific surface area of $\mathrm{TiO}_{2}$ blocking layer, which is normally in a rather compact form. Since this could lead to increase in surface area of the neighboring $\mathrm{TiO}_{2} \mathrm{SC}$ layer, we anticipated a higher dye loading on $\mathrm{TiO}_{2} \mathrm{SC}$ layer. We also 
expected a favorable widening of band gap for $\mathrm{TiO}_{2}$ in blocking layer with NCB loading. When an electron moves from mesoporous $\mathrm{TiO}_{2}$ layer to TCO, lower potential barrier by this change in band gap could reduce electron loss due to recombination. The goal of this study is to confirm these arguments and to accomplish an improved ECE with $0.0 \sim 0.5 \mathrm{wt} \% \mathrm{NCB}$ loading into $\mathrm{TiO}_{2}$ blocking layer in DSSC.

\section{Experimental Procedure}

NCB was ultra-sonicated in ethanol for $1 \mathrm{hr}$, and dropped on a carbon-coated $\mathrm{Cu}$ grid and dried for microstructural analysis by TEM (H-7600, Hitach Co.) up to a magnification of 30,000. Its chemical composition was analysed by microRaman spectroscopy (UniRaman, UniThink Co.) using NCB dispersed in ethanol on a silicon wafer. Characteristic peaks from carbon allotropes in NCB were identified under exposure for 5 seconds in the range of $1200 \sim 2900 \mathrm{~cm}^{-1}$ in an accumulation mode for 60 scans with noise elimination.

The blocking layer of DSSC was prepared by adding $0.0 \sim 0.5 \mathrm{wt} \%$ NCB (average dia. $=55 \mathrm{~nm}$, DASHBLACK, OCI Co.) into $\mathrm{TiO}_{2}$ layer. The process consists of; (a) dispersing NCB in a solution of Titanium(IV)bis(ethyl aceto acetato)-diisopropoxide and 1-Butanol, (b) ultra-sonication followed by spin-coating for $500 \mathrm{rpm}-10$ seconds and 2000 rpm-40 seconds, and (c) heat-treated at $500^{\circ} \mathrm{C}$ for $15 \mathrm{~min}$.

We observed the blocking layers by an optical microscope (Model 815000, GIA Instruments Co.) under dark-field and overhead spot illuminations. A digital camera (Coolpix 4500, Nikon Co.) attached on eyepiece lens produced the necessary images of the blocking layers.

AFM (SPM25DRM, Park Scientific Instruments) was used to confirm the increase in specific surface area of $\mathrm{TiO}_{2}$ layer with NCB addition (0 wt\% and $0.5 \mathrm{wt} \%$ ) after coating the layer on a glass substrate. Surface roughness was measured in terms of RMS (root mean square) by scanning over $2 \times 2 \mu \mathrm{m}^{2}$ area in a non-contact mode.

Band gap changes of NCB-added (0 wt\% and $0.5 \mathrm{wt} \%)$ blocking layer were confirmed by measuring transparency in the wavelength range of $300 \sim 800 \mathrm{~nm}$ at medium scan speed with UV-VIS-NIR (Shimadzu Co., UV3105PC), followed by Tauc plot transformation (Tauc plot program, Shimadzu Co.).

$\mathrm{TiO}_{2} \mathrm{SC}$ film was doctor-bladed on the prepared blocking layer by using $\mathrm{TiO}_{2}$ paste ( $20 \mathrm{~nm}$, Dyesol DSL $18 \mathrm{NR}-\mathrm{T}$ of 10 ) and heat-treated at $500^{\circ} \mathrm{C}$ for $30 \mathrm{~min}$. Dye was adsorbed on $\mathrm{TiO}_{2} \mathrm{SC}$ by using $0.5 \mathrm{mM}$ of cis-vis bis-ruthenium (II) bistetrabutylammonium (N719). The multi-layered working electrode was completed as shown bottom of Fig. 1, and it consists of a glass, FTO (fluorine-doped tin oxide), blocking layer $\left(\mathrm{TiO}_{2}\right.$ with $\left.\mathrm{NCB}\right), \mathrm{TiO}_{2}$ semiconductor (SC), and dye (N719).

The counter electrode was prepared by RF sputter (MHS1500, Moohan Co., $300 \mathrm{~W}, 13.56 \mathrm{MHz}$ ) to form a $100 \mathrm{~nm}-\mathrm{Pt}$ film on a glass substrate with a target of $99.99 \%$ Pt. A flow of $40 \mathrm{sccm}$ Ar at pressure of 5 mtorr at room temperature (RT) was set for the process. The prepared working and counter electrodes were fixed at position and filled with electrolyte. Fig. 1 shows the final version of DSSC consisted of working electrode/electrolyte/counter electrode. Its active area was estimated as $0.45 \mathrm{~cm}^{2}$.

Impedance of DSSC was determined by solar simulator (PEC-L11, Peccell) and potentiostat (Iviumstat, Ivium) to verify interfacial resistance. The analysis carried out in the frequency range of $10 \mathrm{mHz} \sim 1 \mathrm{MHz}$ applying $\mathrm{AC}$ voltage and collecting the current responses. I-V (current-voltage) characteristic of DSSC was measured by the same instruments, but under a different setup; a $100 \mathrm{~W}$ Xenon lamp was the illumination source at 1 sun $\left(100 \mathrm{~mW} / \mathrm{cm}^{2}\right)$ condition. From the I-V curves, short-circuit current density, open circuit voltage, fill factor, and ECE were evaluated.

\section{Results and Discussion}

Figure 2 shows TEM image of NCB magnified to 30,000 times, verifying an average particle size of $55 \mathrm{~nm}$ and revealing an agglomerated, spherical, and amorphous form. No crystalline allotrope such as $\mathrm{CNT}$ or $\mathrm{C}_{60}$ was observed, thus we suggested the presence of graphite and/or graphene as the main phases in NCB.

Figure 3 is the result of Raman analysis for NCB which indicates the presence of pure graphite only. Although peaks at $1350 \mathrm{~cm}^{-1}$ and $1600 \mathrm{~cm}^{-1}$ are for both graphite and graphene, the presence of graphene is excluded since

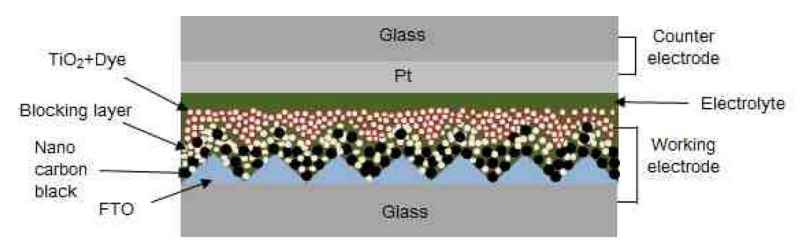

Fig. 1. Illustration of the cross sectional structure of the proposed DSSC.

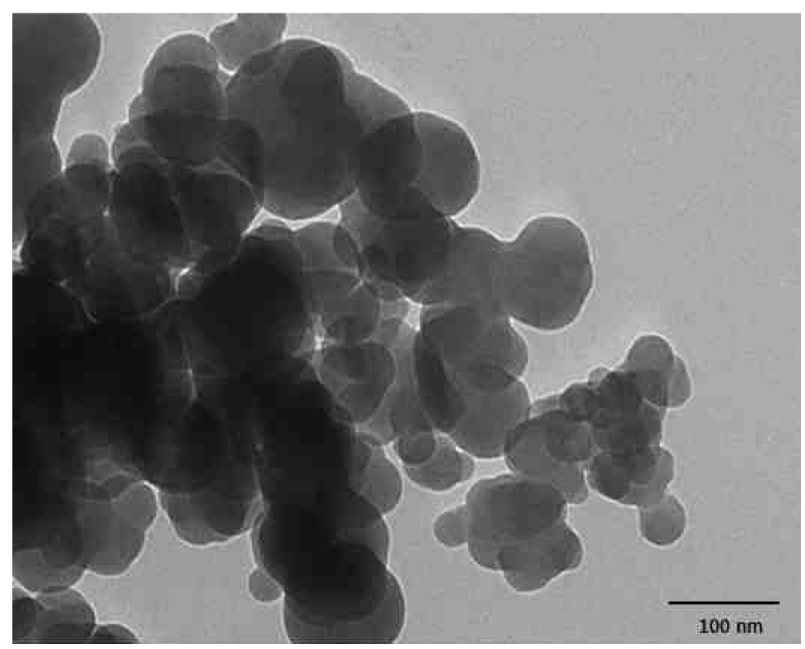

Fig. 2. TEM image of nano carbon black. 
another strong peak at $2700 \mathrm{~cm}^{-1}$ for graphene is absent. ${ }^{7,9)}$

Figure 4 shows optical images of electrodes without and with the dispersed $0.5 \mathrm{wt} \%$ NCB in the blocking layer. By comparing with Fig. 4(a), we confirmed that shinny contrast spots in Fig. 4(b) represent evenly-dispersed NCB. It also suggests that ultra sonicator and spin coating are appropriate processes to load NCB uniformly.

Figure 5 is AFM images of the blocking layers on a glass substrate with $0.0 \mathrm{wt} \%$ and $0.5 \mathrm{wt} \% \mathrm{NCB}$, which show RMS values of $2.5 \mathrm{~nm}$ and $6.41 \mathrm{~nm}$, respectively. Thus, we verified increase in specific surface area of blocking layer by adding NCB on the blocking layer.

Figure 6 is a Nyquist diagram composed of real and imaginary terms for DSSCs with $0.0 \sim 0.5 \mathrm{wt} \%$ NCB under applied frequency. It is evident that the curves show three

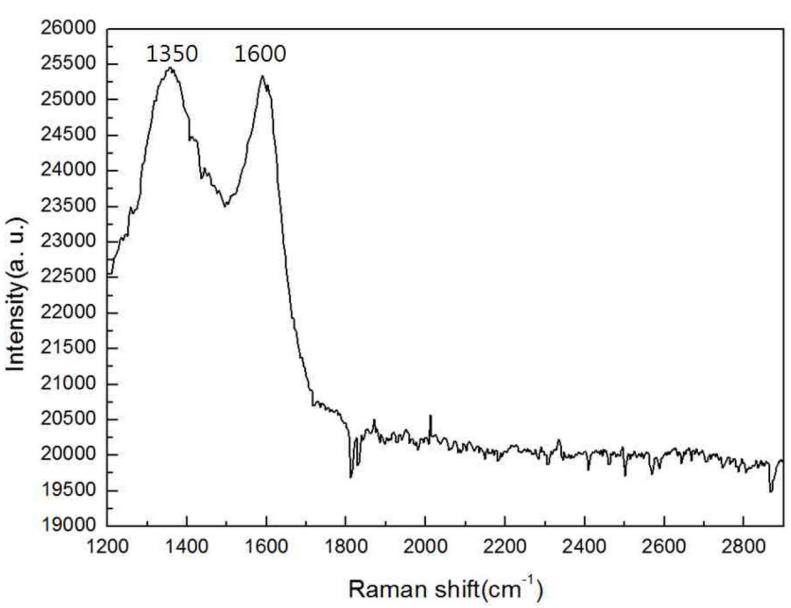

Fig. 3. Raman spectrum of nano carbon black.

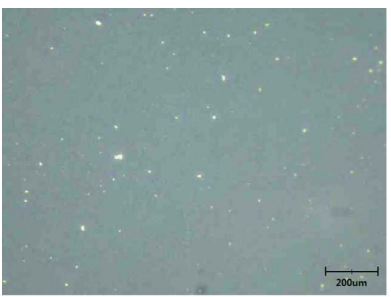

(a)

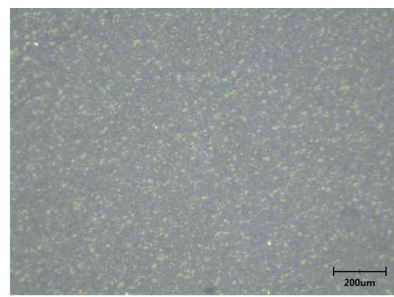

(b)
Fig. 4. Optical images of blocking layers with nano carbon black (NCB): (a) $0.0 \mathrm{wt} \% \mathrm{NCB}$, and (b) $0.5 \mathrm{wt} \% \mathrm{NCB}$.

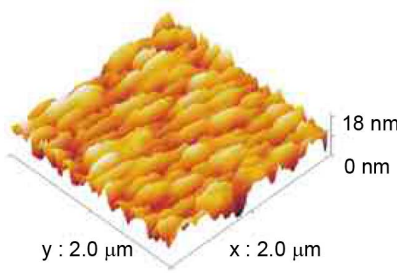

(a)

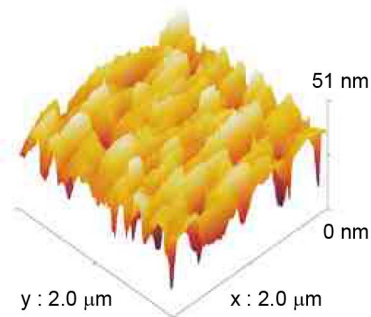

(b)
Fig. 5. AFM images of blocking layer with nano carbon black NCB: (a) $0.0 \mathrm{wt} \%$ NCB, and (b) $0.5 \mathrm{wt} \%$ NCB. half-circle $\left(R_{1}, R_{2}, R_{3}\right)$ like an internal resistance curve of conventional DSSC. $R_{1}$ value at $10^{3} \sim 10^{5} \mathrm{~Hz}$ represents interfacial resistance by electron transport across $\mathrm{TCO} / \mathrm{TiO}_{2}$ and electrolyte/counter electrode. The value decreased drastically from $14.6 \Omega$ to below $2.0 \Omega$ with NCB addition. It is attributed to increased contacts between TCO and $\mathrm{TiO}_{2}$ layer originated from increased surface area of $\mathrm{TiO}_{2}$ layer with NCB loading.

$R_{2}$ value at $1 \sim 10^{3} \mathrm{~Hz}$ represents resistance within $\mathrm{TiO}_{2}$ $\mathrm{SC}$ and across $\mathrm{TiO}_{2}$ SC/electrolyte interface. $\mathrm{R}_{2}$ values are all about $5.5 \Omega$, since the involved components are same. $R_{3}$ value at higher than $10^{6} \mathrm{~Hz}$ is related to diffusing redox species within the electrolyte. $R_{3}$ values are all about $3 \Omega$, since the electrolyte is same. Thus, we confirmed that NCB addition increased specific surface area of $\mathrm{TiO}_{2}$ layer, and decreased interfacial resistance between $\mathrm{TCO}$ and $\mathrm{TiO}_{2}$ layer.

Figure 7 is I-V data of DSSC with $0.0 \sim 0.5$ wt\% NCB addition. NCB addition clearly increased short-circuit cur-

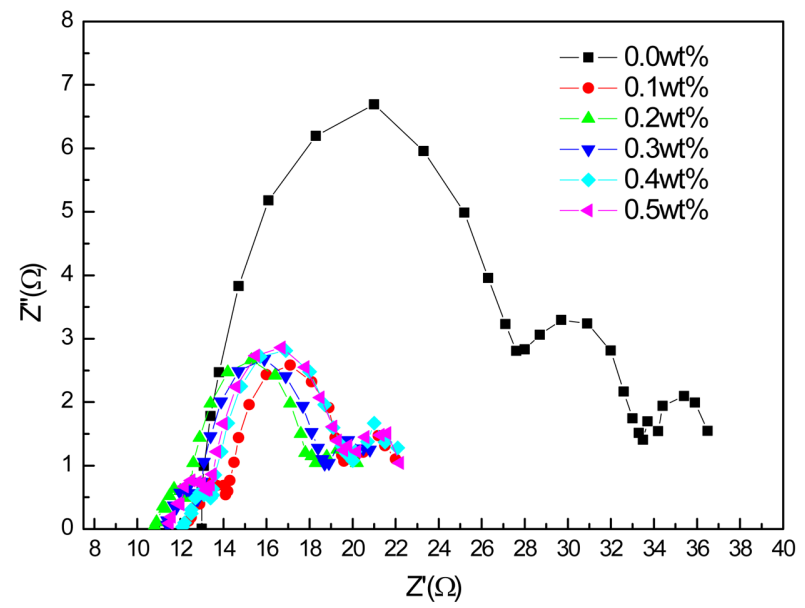

Fig. 6. Impedance of DSSCs employing blocking layer in which the amount of nano carbon blacks varies in the range of $0.0 \sim 0.5 \mathrm{wt} \%$.

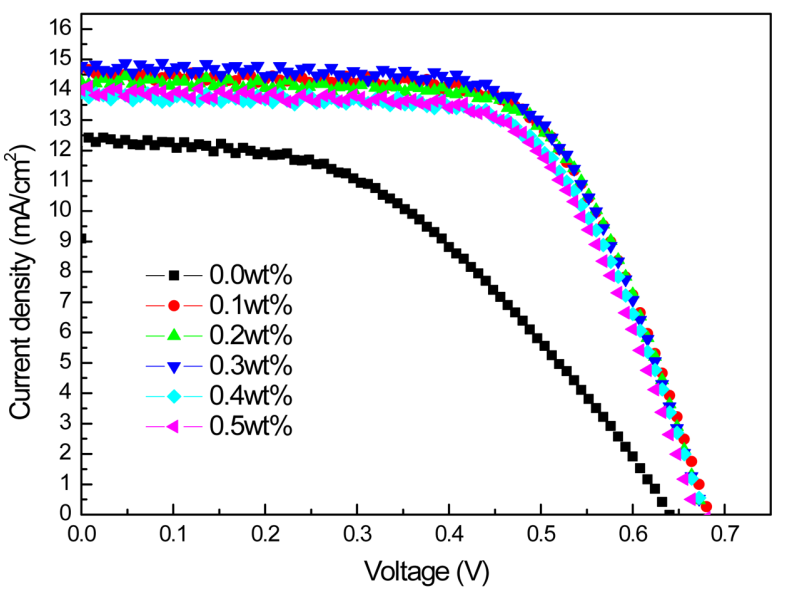

Fig. 7. Current-voltage (I-V) characteristic of DSSCs employing blocking layer in which the amount of nano carbon blacks varies in the range of $0.0 \sim 0.5 \mathrm{wt} \%$. 
rent density, open circuit voltage, and fill factor, and thus increased ECE.

Table 1 is a summary of results for I-V curves of Fig. 7. NCB addition of $0.3 \mathrm{wt} \%$, especially, increased ECE up to $6.20 \%$, which corresponds to enhancement of 1.75 times. We believe that increase in surface area and change within the band gap by NCB addition are responsible for the result.

Firstly, this argument supported by the surface roughness measurement as shown in Fig. 5 indicates that increase in surface area of blocking layer can induce the same effect on the mesoporous $\mathrm{TiO}_{2}$ above, and eventually can give a higher dye absorption. Also, impedance results as shown in Fig. 6 and the decrease in value of $R_{1}$, which is interfacial resistance between $\mathrm{TCO}$ and $\mathrm{TiO}_{2}$, indicate increase in specific surface area. Thus, the result showed reduction in the value of $\mathrm{R}_{1}$ as both specific surface area of mesoporous $\mathrm{TiO}_{2}$, which was formed on the blocking layer and adsorption capacity increased at the same time according to increased specific surface area of the blocking layer itself by adding NCB on the blocking layer. Meanwhile, decreasing ECE with excessive NCB ( $>0.4 \mathrm{wt} \%$ ) can be understood by the same argument; with over-loaded NCB, the effective path for electron transport was rather decreased due to the relatively-reduced portion of $\mathrm{TiO}_{2}$ in the blocking layer that is responsible for the electron move.

Secondly, the possibility of increase in ECE by band gap change according to addition of NCB can be verified from the values of short-circuit current density and open circuit voltage as shown in Table 1 . Short-circuit current density increased certainly by addition of NCB. This means the possibility for formation of new band which reduces recombination during electron transport across existing $\mathrm{TiO}_{2} \mathrm{SC}$ layer to blocking layer. Also, the possibility can be verified by increase in open circuit voltage which is related to the Fermi level of $\mathrm{TiO}_{2}$ electrode. Minor increase in band gaps is verified according to $\mathrm{NCB}$ addition as we obtained more direct evidence from UV-VIS-NIR spectroscopy that shows band gaps of 3.60 and $3.61 \mathrm{eV}$ with $0.0 \mathrm{wt} \%$ and $0.3 \mathrm{wt} \%$ NCB additions, respectively. We decided that the increase in band gaps reduces electron loss when produced electrons from the dye move to the blocking layer from mesoporous $\mathrm{TiO}_{2}$ which is oxide $\mathrm{SC}$ layer.

In summary, appropriate amount of NCB addition resulted in an increase in specific surface area both on

Table 1. Photovoltaic Performance and Energy Conversion Efficiency of DSSC

\begin{tabular}{ccccc}
\hline Sample & Voc $(\mathrm{V})$ & FF & Jsc $\left(\mathrm{mA} / \mathrm{cm}^{2}\right)$ & $\eta(\%)$ \\
\hline 0.0 wt\% & 0.632 & 0.437 & 12.81 & 3.53 \\
0.1 wt\% & 0.689 & 0.616 & 14.73 & 6.10 \\
0.2 wt\% & 0.682 & 0.632 & 14.24 & 6.11 \\
0.3 wt\% & 0.682 & 0.621 & 14.78 & 6.20 \\
0.4 wt\% & 0.684 & 0.620 & 13.92 & 5.80 \\
0.5 wt\% & 0.675 & 0.616 & 13.98 & 5.74 \\
\hline
\end{tabular}

blocking layer and on $\mathrm{TiO}_{2} \mathrm{SC}$ layer. We also created a slightly wider band gap which provides a reduced electron loss during electron transport, and successfully enhanced ECE of DSSC.

\section{Conclusions}

We employed $0.0 \sim 0.5 \mathrm{wt} \%$ of NCB into the blocking layers of working electrode of DSSC, and evaluated their physical and opto-electrical properties. NCB increased the specific surface area of blocking layer and decreased the interfacial resistance between $\mathrm{TCO}$ and $\mathrm{TiO}_{2}$. We also verified that the ECE increases up to $0.3 \mathrm{wt} \%$ addition of $\mathrm{NCB}$. This attributes to the increase in specific surface area of blocking layer and to the newly-formed band of which can decrease interfacial resistance. However, when more than $0.4 \mathrm{wt} \%$ of NCB was added, the efficiency decreased due to the decrease in short-circuit current density coming from the diminished $\mathrm{TiO}_{2}$ portion which is responsible for electron transport. By employing the appropriate amount of NCB, we demonstrated an improvement on ECE of DSSC device.

\section{Acknowledgments}

This paper was supported by Basic Science Research Program through the National Research Foundation of Korea( NRF) funded by the Ministry of Education, Science and Technology (grant number 2011-0006629).

\section{REFERENCES}

1. B. O'Regan and M. Grätzel, "A Low-Cost, High-Efficiency Solar Cell Based on Dye-Sensitized Colloidal $\mathrm{TiO}_{2}$ Films," Nature, 353737 (1991).

2. M. K. Nazeeruddin, A. Kay, R. Humpbry-Baker, E. Miiller, P. Liska, N. Vlachopoulos, and M. Gratzel, "Conversion of Light to Electricity by Cis-X2bis (2,2'-bipyridyl-4,4'-dicarboxylate) Ruthenium(II) Charge-Transfer Sensitizers (X = Cl-, Br-, I-, CN-, and SCN-) on Nanocrystalline Titanium Dioxide Electrodes," J. Am. Chem. Soc., 115 [14] 6382-90 (1993).

3. B. Kılıç, N. Gedik, S. P. Mucur, A. S. Hergul, and E. Gür, "Band Gap Engineering and Modifying Surface of $\mathrm{TiO}_{2}$ Nanostructures by $\mathrm{Fe}_{2} \mathrm{O}_{3}$ for Enhanced-Performance of Dye Sensitized Solar Cell," Mater. Sci. Semicond. Process., 31 363-71 (2015).

4. S. Zhang, H. Niu, Y. Lan, C. Cheng, J. Xu, and X. Wang, "Synthesis of $\mathrm{TiO}_{2}$ Nanoparticles on Plasma-Treated Carbon Nanotubes and its Application in Photoanodes of DyeSensitized Solar Cells," J. Phys. Chem. C, 115 22025-34 (2011).

5. D. Y. Kim, J. Kim, J. Kim, A. Kim, G. Lee, and M. Kang, "The Photovoltaic Efficiencies on Dye Sensitized Solar Cells Assembled with Nanoporous Carbon/ $/ \mathrm{TiO}_{2}$ Composites," J. Ind. Eng. Chem., 18 1-5 (2012).

6. Y. Noh, K. Kim, M. Choi, and O. Song, "Properties of Work- 
ing Electrodes with Nano Diamond Addition in a Dye Sensitized Solar Cell (in Korean)," Korean J. Met. Mater., in press (2015).

7. M. Pawlyta, J. Roudzaud, and S. Duber, "Raman Microspectroscopy Characterization of Carbon Black: Spectral Analysis and Structural Information," Carbon, 84 479-90 (2015).

8. C. Ting and W. Chao, "Efficiency Improvement of the DSSCs by Building the Carbon Black as Bridge in Photoelectrode," Applied Energy, 87 2500-05 (2010).

9. D. Yoon, H. Moon, H. Cheong, J. S. Choi, J. A. Choi, and B.
H. Park, "Variations in the Raman Spectrum as a Function of the Number of Graphene Later," J. Korean Phys. Soc., 55 1299-303 (2009).

10. K. Lim, Y. Kim, S. Kim, and I. Han, "Effect of Carbon Source on Porosity and Flexural Strength of Porous SelfBonded Silicon Carbide Ceramics," J. Korean Ceram. Soc., 45 [7] 430-37 (2008).

11. J. Eom, Y. Kim, and I. Song, "Effect of SiC Filler Content on Microstructure and Flexural Strength of Highly Porous SiC Ceramics Fabricated from Carbon-Filled Polysiloxane," J. Korean Ceram. Soc., 49 [6] 625-30 (2012). 